\title{
Small Lymphocytic Lymphoma with Plasmacytoid Differentiation
}

National Cancer Institute

\section{Source}

National Cancer Institute. Small Lymphocytic Lymphoma with Plasmacytoid

Differentiation. NCI Thesaurus. Code C36273.

A small lymphocytic lymphoma characterized by the presence of neoplastic lymphocytes with plasmacytoid morphology. 